\title{
Under-Sampling and Compressed Sensing of 3D Spatially- Resolved Displacement Propagators in Porous Media using APGSTE-RARE MRI
}

Daan W. de Kort ${ }^{\mathrm{a}}$, Stefan A. Hertel ${ }^{\mathrm{b}}$, Matthias Appel ${ }^{\mathrm{c}}$, Hilko de Jong ${ }^{\mathrm{b}}$, Michael D. Mantle ${ }^{\mathrm{a}}$, Andrew J. Sederman ${ }^{\mathrm{a}}$, Lynn F. Gladden ${ }^{\mathrm{a},{ }^{*}}$

a) Magnetic Resonance Research Centre, Department of Chemical Engineering and Biotechnology, University of Cambridge, Philippa Fawcett Drive, Cambridge CB3 OAS, United Kingdom

b) Shell Technology Center Houston, Shell Exploration and Production Inc., 3333 Highway 6 S, Houston, TX 77082, USA

c) Shell Technology Centre Amsterdam, Shell Global Solutions International B.V., Grasweg 31, 1031 HW Amsterdam, The Netherlands

* Corresponding author

$\begin{array}{ll}\text { Name: } & \text { Lynn F. Gladden } \\ \text { Email: } & \text { lfg1@ @am.ac.uk }\end{array}$

Tel: $\quad$ (+44) 1223334762

Revision submitted: 26 August 2018 


\begin{abstract}
A method for under-sampling and compressed sensing of 3D spatially-resolved propagators is presented and demonstrated for flow in a packed bed and a heterogeneous carbonate rock. By sampling only $12.5 \%$ of $\mathbf{q}, \mathbf{k}$-space, the experimental acquisition time was reduced by almost an order of magnitude. In particular, for both systems studied, a 3D image was acquired at $1 \mathrm{~mm}$ isotropic spatial resolution such that 134,400 local propagators were obtained. Data were acquired in $\sim 1 \mathrm{~h}$ and $\sim 11 \mathrm{~h}$ for the packed bed and rock, respectively. It is shown that spatial resolution and under-sampling using this implementation retains the quantitative nature of the propagator measurement, and differences between implementation of this measurement in two and three dimensions are identified. The potential for 3D spatially-resolved propagators to provide new insights into transport processes in porous media by characterisation of the statistical moments of the propagators is discussed.
\end{abstract}

Keywords: spatially-resolved propagators; flow; porous media 


\section{Introduction}

Pulsed field gradient (PFG) magnetic resonance imaging (MRI) is an invaluable tool for understanding fluid transport through porous media. It can be used to spatially map average fluid velocities throughout a porous medium of interest [1], or, alternatively, to spatially resolve ensembleaverage displacement propagators [2-4]. Although spatially-resolved propagators provide a comprehensive characterisation of fluid motion, which includes contributions from flow, dispersion and self-diffusion, their measurement is costly in terms of experimental time, because images need to be acquired for a range of q-values. Alternatively, a velocity map can be derived from only two images acquired at different q-values, which constitutes a much quicker measurement, although with this methodology accuracy of the flow measurement may be compromised if there is a high degree of asymmetric dispersion in the local flow field [5]. To reduce data acquisition times for spatiallyresolved propagators, it is possible to acquire only a subset of data points in $\mathbf{q}, \mathbf{k}$-space. However, direct Fourier transformation of under-sampled data leads to image artefacts due to violation of the Nyquist sampling rate. It has previously been shown that compressed sensing (CS) can be used for artefact-free recovery of magnetic resonance data $[6,7]$ and recently, application to the measurement of a 2D spatially-resolved propagator from under-sampled $\mathbf{q}, \mathbf{k}$-data has been demonstrated [2]. In this paper the extension of this work to include a third spatial dimension is described. The method is then demonstrated with respect to the acquisition of a 3D spatially-resolved propagator of flow through a packed bed of spheres and hollow cylinders, and a heterogeneous carbonate rock core.

As is well known, displacement propagators are probability distributions of molecular displacements $\bar{P}(\mathbf{r}, \Delta)$, where $\bar{P}$ is the probability that a spin moves over a distance $\mathbf{r}$ in an observation time $\Delta$. Pulsed-field gradient (PFG) NMR is routinely used to acquire spatially-unresolved displacement

propagators. The signal intensity $A$ is measured as a function of $\mathbf{q}=\frac{\gamma \delta \mathbf{g}}{2 \pi}$, where $\mathbf{g}$ is a vector describing the intensity and direction of the applied gradient pulses, $\delta$ the time for which the pulses are applied, and $\gamma$ the gyromagnetic ratio. $A(\mathbf{q})$ is related to the propagator, for a given $\Delta$, via Fourier transformation (FT):

$\bar{P}(\mathbf{r}, \Delta)=\int A(\mathbf{q}) e^{-2 \pi i \mathbf{q} \cdot \mathbf{r}} \mathrm{d} \mathbf{q}$.

In order to acquire spatially-resolved displacement propagators, the q-encoding PFG NMR experiment needs to be coupled to an imaging experiment to acquire $\mathbf{k}$-space. The spatially-resolved propagator is then obtained by Fourier transformation of the whole $\mathbf{q}, \mathbf{k}$-space. 
The results presented here extend recent work in which a 13-interval Alternating Pulsed Gradient Stimulated Echo (APGSTE) PFG NMR experiment [8] was combined with a Rapid Acquisition with Relaxation Enhancement (RARE) MRI experiment [9] for spatially resolving the displacements to produce spatially-resolved propagators in $2 \mathrm{D}$ slice sections within a porous medium. In the present work, a 4-dimensional q,k-space (q-space 1-dimensional, $\mathbf{k}$-space 3-dimensional) is sampled according to a quasi-random under-sampling pattern so that the propagators are spatially resolved in all 3 spatial dimensions. The propagators, which are encoded in $\mathbf{q}$, can be measured in any arbitrary spatial direction. The under-sampling APGSTE-RARE MRI experiment imposes some boundary conditions on the sampling pattern, but as will be outlined below, it can accommodate essentially any degree of sampling and imposes no restrictions on the position in $\mathbf{k}_{\text {phase }}$ of the points sampled. In contrast to the 2D spatially-resolved propagators, in which a phase-encoded, a frequency-encoded, and a slice-selective dimension were used for spatial encoding, for the 3D spatially-resolved case there is no slice-selective dimension, but an additional phase-encoded dimension. The additional phase-encoded dimension can be under-sampled, allowing for greater freedom in the design of the sampling pattern relative to the 2D spatially-resolved case.

\section{Experimental design}

In this section, the experimental acquisition of under-sampled 3D spatially-resolved propagators is described. First, the APGSTE-RARE pulse sequence used to acquire the 4-dimensional undersampled q,k-data is described. Second, the under-sampling scheme used in combination with this experiment is shown. Differences between acquisitions of 3D and 2D spatially-resolved propagators are highlighted.

\subsection{APGSTE-RARE experiment}

A schematic of the pulse sequence used to acquire 3D spatially-resolved propagators is shown in Fig. 1. As described in our earlier work [2], the 13-interval APGSTE sequence was used for displacement (q) encoding, because it partially compensates for the effects of internal gradients that are expected to be present in many of the porous media of interest [8]. The RARE experiment was used for spatial (k) encoding. In the 3D spatially-resolved RARE experiment, one dimension, $\mathbf{k}_{\text {read }}$, is frequency encoded, and two dimensions, $\mathbf{k}_{\text {phase }_{1}}$ and $\mathbf{k}_{\text {phase }_{2}}$, are phase encoded. Throughout this work, displacements were measured along $z$-direction, i.e., $\mathbf{q}=\mathbf{q}_{z}$, parallel to $\mathbf{k}_{\text {read }}$. For the acquisition of 3D spatiallyresolved propagators, all r.f. pulses were hard and rectangular, and a second phase-encoded dimension replaced the slice-selective dimension used in the 2D spatially-resolved case. Spatially-resolved propagators were acquired employing XY phase cycling [10] rather than conventional CPMG phase cycling $[11,12]$ on the RARE inversion pulse train . It has been shown previously that the application of an XY phase cycle, and the resulting need to acquire individual, identical data sets in the odd and 
even echoes that are recombined during post-processing, has no significant influence on the quality of propagator measured on packed beds and rock samples [2,13]. However, due to the need to acquire individual data sets in the odd and even echoes, the factor by which the experimental time is reduced does not equal the number of echoes $\left(N_{\text {ech }}\right)$ as in conventional RARE, but rather $1 / 2 N_{\text {ech. }}$.

\subsection{Sampling pattern design}

For the case of an APGSTE-RARE acquisition of 3D spatially-resolved propagators, three of the four data dimensions can be under-sampled. Only the readout dimension, $\mathbf{k}_{\text {read }}$, is always fully sampled, which does not come at a significant cost in terms of acquisition time. For the $\mathbf{k}_{\text {phase }}$ and $\mathbf{q}$ dimensions, the sampling scheme employed follows that used in previous work $[2,14,15]$. To minimise coherent artefacts in the CS reconstructions due to under-sampling, pseudo-random patterns are generated on the basis of a polynomial probability density function (pdf) [16]:

$\operatorname{pdf}=(1-r)^{n}$,

where $r \in 0<r<1$ is a measure of distance from the centre of reciprocal space. The central point in $\mathbf{q}, \mathbf{k}$-space and a finite region around the central point, where the amplitude of the Fourier coefficients is particularly high, is always sampled fully. The choice of exponent $n$ influences the CS reconstruction quality, and is typically chosen such that the shape of the pdf qualitatively resembles the Fourier amplitude distribution in reciprocal space.

The potentially different distribution of the intensities of the Fourier coefficients in the $\mathbf{q}$ and $\mathbf{k}$ dimensions was adjusted for by varying exponent $n$ of the pdf in Eq. 2 cyclically between the two phase encoded dimensions, $\mathbf{k}_{\text {phase }_{1}}$ and $\mathbf{k}_{\text {phase }_{2}}$, and the $\mathbf{q}$ dimension as:

$n_{\mathbf{q}, \mathbf{k}_{\text {phase }}}=n_{\mathbf{q}}+\left(n_{\mathbf{k}_{\text {phase }}}-n_{\mathbf{q}}\right)\left(\frac{\left|\mathbf{k}_{\text {phase }_{1}}+\mathbf{k}_{\text {phase }_{2}}\right|}{\left|\mathbf{k}_{\text {phase }_{1}}+\mathbf{k}_{\text {phase }_{2}}+\mathbf{q}\right|}\right)^{2}$,

where $\mathbf{k}_{\text {phase }_{1}}, \mathbf{k}_{\text {phase }_{2}}$ and $\mathbf{q}$ are vectors, and $n_{\mathbf{k}_{\text {phase }}}$ and $n_{\mathbf{q}}$ are the exponents along the $\mathbf{k}_{\text {phase }}$ and $\mathbf{q}-$ directions, respectively. A single value for $n_{\mathbf{k}_{\text {phase }}}$ is used for both phase directions because the packed bed and rock core used in this work are structurally isotropic with respect to these directions. Constraints imposed on the under-sampling scheme generated on the basis of this pdf are the same as in the case of 2D spatially-resolved propagators [2].

Figure 2 shows the sidelobe-to-peak ratios (SPR) for a range of different pseudo-random $\mathbf{q}, \mathbf{k}_{\text {phase }}{ }^{-}$ sampling patterns, which are calculated from their density-compensated point-spread functions [16], 
for different values of $N_{\text {ech }}$ and degrees of sampling. It is of interest to note that, in contrast to similar data presented for the acquisition of 2D spatially-resolved propagators [2], the SPR is not as sensitive to $N_{\text {ech. }}$. The reason is that at higher $N_{\text {ech }}$, the impact of the constraint that the number of $\mathbf{k}_{\text {phase }}$-points sampled per point in q should always be a multiple of $1 / 2 N_{\text {ech }}$ becomes lower, because those points can be accommodated in the additional dimension in a more incoherent fashion. The order in which the $\mathbf{k}_{\text {phase }}$ points in the sampling pattern are acquired experimentally within the RARE echo train is governed by their distance to the centre of $\mathbf{k}$-space, as described by Ramskill et al. [14].

\subsection{Data reconstruction}

\subsubsection{Fully-sampling and Fourier transformation}

As in the case of 2D spatially-resolved propagators [2], for the case of fully-sampling, the data were Fourier-transformed in the k- and q-dimensions. As described in that work, data from the odd and even echoes were separated, and either one was flipped in the q-dimension. A zeroth-order phase correction was applied according to the central point in $\mathbf{q}, \mathbf{k}$-space. The data from the odd and even echoes were then recombined. A window function was then applied in all $\mathbf{k}$-dimensions in order to suppress truncation artefacts due to the presence of sharp interfaces in the spatial domain, which also affect the quantitative nature of the per-voxel propagators. Because the window function is not applied in the q-dimension, the propagators themselves are not broadened. In the case of processing the 3D spatially-resolved propagators, a sine window, as used in the 2D spatially-resolved case, was found not to be powerful enough to suppress the truncation artefacts. Instead, a Gaussian window function is used such that if the central point of $\mathbf{k}$-space is sampled at the point with index $m=m_{\mathbf{k}_{0}}$, the Gaussian window $w$ in each $\mathbf{k}$-dimension is defined as:

$w(m)=e^{-\frac{1}{2}\left(\frac{m-m_{\mathbf{k}_{0}}}{M m_{\mathbf{k}_{0}}}\right)^{2}}$,

where $M$ is a positive number. The standard deviation of the window equals $M m_{\mathbf{k}_{0}}$ lattice units of width $\Delta \mathbf{k}$. It was found that adequate suppression of the truncation artefacts was achieved by using $M \geq 0.5$. As a final step, after Fourier transformation, the resulting spatially-resolved propagator was phase corrected in first order.

\subsubsection{Under-sampling and compressed sensing reconstruction}

For the case of under-sampled data, two methods of reconstruction were implemented: zero-filling prior to Fourier transformation, and compressed sensing reconstruction using total variation (TV) regularisation. The latter reconstruction has been described elsewhere [2], and is summarised as follows. Let $\boldsymbol{u}$ denote a spatially-resolved propagator, $\mathcal{F}$ the Fourier-transform operator, and $S$ the 
sampling pattern; the under-sampled (noisy) data $\boldsymbol{y}$ and the (noisy) spatially-resolved propagator $\boldsymbol{u}$ are then related through:

$\boldsymbol{y}=S \mathcal{F} \boldsymbol{u}$.

Compressed sensing employs prior knowledge about $\boldsymbol{u}$ to find a better solution, which is denoted $\boldsymbol{u}_{\mathrm{CS}}$. This prior knowledge relates to $\boldsymbol{u}$ having a sparse representation in a certain transform domain. Using this knowledge, a solution for $\boldsymbol{u}_{\mathrm{CS}}$ is determined subject to the optimisation:

$\boldsymbol{u}_{\mathrm{CS}} \in \arg \min _{\boldsymbol{u}}\left\{\frac{1}{2}\|S F \boldsymbol{u}-\boldsymbol{y}\|_{2}^{2}+\alpha J(\boldsymbol{u})\right\}$

where $\frac{1}{2}\|S \mathcal{F} \boldsymbol{u}-\boldsymbol{y}\|_{2}^{2}$ is a fidelity term that ensures consistency with the acquired data, and $\alpha J(\boldsymbol{u})$ is a Tikhonov-regularisation term. $J(\boldsymbol{u})$ is a regularisation functional by which a measure of the transform sparsity of $\boldsymbol{u}$ is incorporated into the optimisation problem and the choice of $J(\boldsymbol{u})$ is based on the compressibility of $\boldsymbol{u}$ in that transform domain. The fidelity and regularisation terms are balanced by the regularisation parameter $\alpha$, which is always a positive number. In this paper, total variation regularisation (TV) is used for the reconstruction of 3D spatially-resolved propagators, as this was found to be an effective regulariser for 2D spatially-resolved propagators [2]; TV regularisation is based on finite-differencing of $\boldsymbol{u}$, and is defined as:

$J(\boldsymbol{u})=T V(\boldsymbol{u})=\|\nabla \boldsymbol{u}\|_{2,1}$,

where $\|\nabla \boldsymbol{u}\|_{2,1}$ is the $\ell_{1}$-norm of the $\ell_{2}$-norm of the finite-difference approximation of the local gradient $\nabla \boldsymbol{u}$, with zero Neumann boundary conditions. In this application, the $\ell_{2}$-norm of the local gradient, $\|\nabla \boldsymbol{u}\|_{2}$, is calculated isotropically (i.e. in all four of the $\mathbf{k}$ and $\mathbf{q}$ dimensions), as in our previous work [2], as it was found to be an effective regulariser for both the $\mathbf{k}$ and $\mathbf{q}$ domains. An appropriate estimate for regularisation parameter $\alpha$ was chosen on the basis of Morozov's discrepancy principle [17]. Before reconstruction, as for the fully-sampled case, a Gaussian window (Eq. 4) was applied to the under-sampled data to counteract truncation artefacts at sharp interfaces in the spatial domain. The CS reconstructions were then performed using a primal-dual hybrid gradient method as described in detail elsewhere [18-20].

\section{Material and methods}

\subsection{Experimental setup}

Packed bed of spheres and hollow cylinders. As a simple model system for a heterogeneous rock core, a cylindrical random-packed bed was prepared consisting of polyoxomethylene (POM) spheres 
(Simply Bearings Ltd) and hollow cylinders (Bülte Plastics). The use of POM, which has a relatively small difference in magnetic susceptibility relative to water [21], minimises the magnetic field gradients within the bed, which have an adverse effect on the NMR experiments. The bed was packed with an approximately 1:1:1 mix of spheres of $4.0 \mathrm{~mm}$ diameter, and cylinders of two different lengths, 4.0 and $6.0 \mathrm{~mm}$, the outer and inner diameter of both being 4.0 and $2.6 \mathrm{~mm}$, respectively. The internal diameter and length of the bed were $37 \mathrm{~mm}$ and $69 \mathrm{~mm}$, respectively. The total porosity, determined from the cylindrical volume of the bed, the total mass of the POM elements, and the density of POM $\left(1.41 \mathrm{~g} \mathrm{~cm}^{-3}\right)$, was $54.0 \pm 1.0 \%$. Uniform flow at the entrance and exit was promoted by using porous polyethylene distributor plates (SPC Technologies Ltd) at either end of the bed. After loading the elements into the cylindrical column, the packed bed was imbibed with deionised water through vacuum saturation. The packed bed was connected into a closed flow loop, and a constant flow rate of water of $10 \mathrm{~mL} \mathrm{~min}^{-1}$ was imposed using a Quizix QX-1500HC dual-cylinder syringe pump.

Edwards Brown carbonate rock. A cylindrical plug of a heterogeneous carbonate rock ("Edwards Brown"; Kocurek Industries Inc.), of diameter and length $38.1 \mathrm{~mm}$ (1.5 in) and $6 \mathrm{~cm}$, respectively, was used. The total porosity, determined gravimetrically by imbibition of deionised water by vacuum saturation was $37.6 \pm 0.4 \%$. The core was then placed in a cylindrical Ergotech PEEK rock core flow cell and a confining pressure of $2 \mathrm{MPa}$ was applied using 3M Fluorinert FC-43, which is NMR silent in typical ${ }^{1} \mathrm{H}$ chemical shift ranges. The cell was contained in a closed-loop system with a backpressure regulator, and the confining pressure was maintained using a Gilson model 307 pump. A constant flow rate of water of $10 \mathrm{~mL} \mathrm{~min}^{-1}$ was imposed using a Quizix QX-1500HC dual-cylinder syringe pump.

\subsection{Magnetic resonance experiments}

All NMR measurements were made using a Bruker BioSpin AV spectrometer in combination with a horizontal-bore superconducting magnet with a static field strength $B_{0}$ of $2 \mathrm{~T}\left(85 \mathrm{MHz}{ }^{1} \mathrm{H}\right.$ resonance frequency) and a birdcage r.f. coil of $60 \mathrm{~mm}$ diameter. An Agilent SGRAD MkIII 205/120/HD triaxial gradient system with a maximum gradient amplitude $g_{\max }$ of $0.107 \mathrm{~T} \mathrm{~m}^{-1}$ in the three orthogonal $x$-, $y$ - and $z$-directions was used. The $z$-direction was defined as the direction of superficial flow through the sample.

\subsubsection{Packed bed of spheres and hollow cylinders}

As a ground truth to benchmark the performance of the CS reconstruction of under-sampled data, a fully-sampled 3D spatially-resolved propagator was acquired for water flow through the packed bed. Rectangular r.f. pulses were used at a nutation frequency of $13 \mathrm{kHz}$. The field-of-view (FOV) in the $\mathbf{k}_{\text {read }}$ dimension was $88.2 \mathrm{~mm}$, and in both $\mathbf{k}_{\text {phase }}$ dimensions $42 \mathrm{~mm}$. The number of points acquired in 
the read dimension was 84 , and in the phase dimensions 40 , thereby giving an isotropic spatial resolution of $1.05 \mathrm{~mm} \times 1.05 \mathrm{~mm} \times 1.05 \mathrm{~mm}$. Thus, local propagators were obtained for each of the 134,400 voxels in the 3D spatially-resolved propagator. The number of echoes in the RARE loop, $N_{\text {ech }}$, was 40 with an echo time, $\tau_{\mathrm{e}}$, of $2.5 \mathrm{~ms}$. For the APGSTE block, the length of a pair of flowencoding gradients $\delta$ was set to $7.4 \mathrm{~ms}$, and a total of 65 q-points were sampled by applying flowencoding gradients between $-9.6 \times 10^{-2}$ and $9.6 \times 10^{-2} \mathrm{~T} \mathrm{~m}^{-1}$ (90\% of $g_{\max }$ ) in equidistant steps; i.e., symmetrically around the centre of $\mathbf{q}$-space, with the $33^{\text {rd }}$ point at $\mathbf{q}=0 \mathrm{~m}^{-1}$. These settings lead to a FOV in the displacement domain of $1.0 \mathrm{~mm}$. Displacements were measured along the superficial flow direction, i.e. $z$-direction. The displacement-observation time $\Delta$ was $200 \mathrm{~ms}$. Four signal averages were used with a time between excitations of $0.9 \mathrm{~s}$, resulting in a total acquisition time of $7 \mathrm{~h}$.

Under-sampled spatially-resolved propagators of the same sample were acquired using the same pulse sequence settings as for the fully-sampled experiment, except that the sampling pattern was generated using the method described in Section 2.2 based on the pdf defined in Eqs. 2 and 3, with $n_{\mathbf{k}_{\text {phase }}}=7$ and $n_{\mathbf{q}}=4$, and with the diameter of the fully-sampled spherical region being at least 4 points in reciprocal space. The generated sampling scheme sampled $12.5 \%$ of all $\mathbf{q}, \mathbf{k}$-space data points. The resulting acquisition time was $50 \mathrm{~min}$ (using 4 averages and a recycle delay $0.9 \mathrm{~s}$ ). The displacementobservation time was again $200 \mathrm{~ms}$, and the spatial resolution remained $1.05 \mathrm{~mm} \times 1.05 \mathrm{~mm} \times 1.05$ mm.

\subsubsection{Edwards Brown carbonate rock}

The same approach as used for the model packed bed was employed except that the number of echoes in the RARE loop $N_{\text {ech }}$ was reduced to 16 , because of the faster $T_{2}$ relaxation characteristics of the fluid in the rock relative to the model system. The FOV for the displacement domain was enlarged to accommodate the larger displacements observed in the rock relative to the model system at the given flow rate and observation time. To this end, in the APGSTE block, the length of a pair of flowencoding gradients $\delta$ was shortened to $4.6 \mathrm{~ms}$, leading to a FOV of $1.6 \mathrm{~mm}$ in the displacement domain. All other experimental settings remained the same. A sampling pattern was generated based on the same pdf as described in Section 3.2.1 but taking into account the lower $N_{\text {ech }}$. By acquiring 8 averages at a recycle delay $1.5 \mathrm{~s}$, the acquisition time for the fully-sampled experiment was $86 \mathrm{~h}$, and for the under-sampled experiment $11 \mathrm{~h}$.

\section{Results and discussion}

As a metric for the quality of the CS reconstruction of the under-sampled data with respect to the Fourier transform of the fully-sampled data, the peak signal-to-noise ratio (PSNR) is used, which is defined as: 
$\operatorname{PSNR}\left(\boldsymbol{u}_{\mathrm{CS}}, \boldsymbol{u}_{\mathrm{FS}}\right)=20 \log _{10}\left(\frac{\max \left(\boldsymbol{u}_{\mathrm{FS}}\right)}{\left\|\boldsymbol{u}_{\mathrm{CS}}, \boldsymbol{u}_{\mathrm{FS}}\right\|_{2}^{2} / N_{\mathbf{q} \mathbf{k}}}\right)$

where $N_{\mathbf{q}, \mathbf{k}}$ is the total number of elements in $\boldsymbol{u}$. PSNR is expressed in units of $\mathrm{dB}$; a higher value is indicative of a better reconstruction quality. For a perfect reconstruction, $\mathrm{PSNR}=\infty \mathrm{dB}$.

Using a truncated training data set obtained from a fully-sampled experiment, it was verified that for 3D spatially-resolved propagators, the dependency of the reconstruction quality on $N_{\text {ech }}$, and hence the dependency on the SPR associated with the underlying the sampling patterns, is not significant for the patterns that form the basis of Fig. 2. This result implied that higher values for $N_{\text {ech }}$ could be used for the experiments on the bead pack than on the carbonate rock to exploit the slower $T_{2}$ relaxation of water in the packed bed, without compromising on reconstruction quality. As an illustration of the sampling patterns used, Fig. 3 shows the one used for the data acquisition on the carbonate rock $\left(N_{\text {ech }}\right.$ $=8$ ). The three cross-sections in Fig. 3a-c are taken through the centre of $\mathbf{q}, \mathbf{k}_{\text {phase }}$-space. In all three cross-sections it can be seen that a region around the central point is fully sampled. In the pattern in Fig. 3a, which represents the central point in q-space, the number of points sampled is a multiple of $1 / 2 N_{\text {ech }}$. In Figure $3 b$ and $c$ it can be seen that the patterns are symmetrical in the centre of $\mathbf{q}$-space, which is necessary to allow recombination of the odd- and even-echo data sets during postprocessing.

For completeness it is further noted that as with the case of 2D spatially-resolved propagators [2], TV regularisation significantly outperforms nuclear norm $(\mathrm{NN})$ regularisation; this is most likely because of the widely different shapes that the propagators take when considering flow in these highly heterogeneous porous systems.

Given the large amount of data (i.e., local propagators) acquired it is necessary to characterise the heterogeneity of these systems using quantitative statistical descriptors. Here we use only the mean displacement $(\mu)$, and standard deviation of the displacement $(\sigma)$, which is given by the square root of the variance of the propagator:

$\mu=\frac{\int \bar{P}(\mathbf{r}, \Delta) \mathbf{r} \mathrm{d} \mathbf{r}}{\int \bar{P}(\mathbf{r}, \Delta) \mathrm{d} \mathbf{r}}$

$\sigma=\sqrt{\frac{\int \bar{P}(\mathbf{r}, \Delta)(\mathbf{r}-\mu)^{2} \mathrm{~d} \mathbf{r}}{\int \bar{P}(\mathbf{r}, \Delta) \mathrm{d} \mathbf{r}}}$ 
where $\mathbf{r}$ is the displacement along the flow-encoded direction over observation time $\Delta$.

\subsection{Spatially-resolved propagators within the packed bed}

The quantitative nature of the acquisition of 3D spatially-resolved propagators within the packed bed is demonstrated in Fig. 4. In Fig. 4a, a comparison is shown between the spatially-unresolved propagator obtained using four different approaches, namely: (1) an APGSTE-based spatiallyunresolved propagator experiment; (2) a fully-sampled, Fourier-transformed 3D spatially-resolved propagator of which all local propagators have been summed; (3) an under-sampled, zero-filled and Fourier-transformed 3D spatially-resolved propagator of which all local propagators have been summed; and (4) an under-sampled, CS-reconstructed 3D spatially-resolved propagator of which all local propagators have been summed. It is seen that the lines associated with (1), (2), and (4) are indistinguishable, whilst the zero-filled FT solution (3) deviates significantly and contains significant artefacts due to violation of the Nyquist sampling rate. This comparison confirms the quantitative nature of the 3D APGSTE-RARE acquisition relative to an established spatially-unresolved acquisition, and furthermore that for the under-sampled experiment, the zero-filled FT solution is inadequate and that CS reconstruction finds a better solution. Indeed, the PSNR of the CSreconstructed spatially-resolved propagator is $48.4 \mathrm{~dB}$, significantly higher than the $34.5 \mathrm{~dB}$ for the zero-filled FT solution.

In Figure 4b-f, the 3D spatially-resolved propagator is explored on the per-voxel level. Figure $4 \mathrm{~b}$ shows an $x, y$-slice through a 3D intensity image which was acquired at three times higher spatial resolution than the spatially-resolved propagator, i.e. $350 \mu \mathrm{m}$ isotropic spatial resolution. In this image, the constituent spheres and cylinders of different lengths and in different orientations, ranging from parallel to orthogonal to the superficial flow direction, are seen. Four locations are indicated with red circles, located at the tip of each arrowhead. The local propagators associated with voxels 1 , 2, 3, and 4 are shown in Fig. 4c, d, e, and f, respectively; in each of these figures a comparison is made between the fully-sampled FT, and the $12.5 \%$ sampled zero-filled FT and compressed sensing results. Figure $4 \mathrm{c}$ shows that the flow within this hollow cylinder, which lies perpendicular to the direction of superficial flow, is characterised by a propagator of Gaussian form centred around a mean displacement $\mu$ of only $\sim 1 \mu \mathrm{m}$ with a standard deviation $\sigma$ of $30 \mu \mathrm{m}$, i.e. the flow is essentially stagnant within the orthogonally aligned hollow cylinder. This is confirmed by calculating the meansquare displacement of water molecules due to self-diffusivity, $\sqrt{2 D \Delta}$, where $D$ is the self-diffusion coefficient $\left(2.3 \times 10^{-9} \mathrm{~m}^{2} \mathrm{~s}^{-1}\right.$ for water at $\left.298 \mathrm{~K}\right)$ and $\Delta=200 \mathrm{~ms}$, which is found to be $30 \mu \mathrm{m}$, in agreement with the propagator data. Voxel 2 (Fig. 4d) lies within a region of high shear rate, adjacent to the interface of three different cylinders, and the propagator is characterised by $\mu=87 \mu \mathrm{m}$ and $\sigma=$ $85 \mu \mathrm{m}$, indicative of significant additional flow dispersion in addition to molecular self-diffusion. Fig. 
4e shows the propagator associated with voxel 3, which lies at the centre of a cylinder which is aligned parallel to the superficial flow direction. This propagator has a broader, top-hat shape, typical of a propagator characterising laminar flow of a Newtonian fluid through a pipe. For this location, $\mu=$ $211 \mu \mathrm{m}$ and $\sigma=123 \mu \mathrm{m}$, showing significant flow dispersion. Finally, Fig. 4f shows the propagator associated with voxel 4 , which lies within a cylinder oriented at an oblique angle relative to the superficial flow direction. For this propagator, a significant (negative) skew rather than a top-hat shape is observed. The associated parameters are $\mu=124 \mu \mathrm{m}$ and $\sigma=56 \mu \mathrm{m}$.

As was the case for the spatially-unresolved result presented in Fig. 4a, for the local propagators in Fig. 4c-f, the fully-sampled FT and under-sampled CS-results are in excellent agreement. For reference, the zero-filled FT results are also shown, which suffer from significant artefacts from under-sampling and do not agree with the fully-sampled FT result, demonstrating the level of enhancement in quality obtained through CS reconstruction of the under-sampled data. Further, the data in Fig. 4e, in particular, show that the distortions to the shape of the propagator when using the zero-filled FT approach leads to propagators which are too distorted to yield useful information on the transport processes occurring.

In order to further demonstrate the quality of the CS reconstructions relative to the fully-sampled (FS) FT result, a voxel-by-voxel comparison of the mean and standard deviation derived from both data sets is shown in Fig. 5. Intensity thresholding was employed to filter out those voxels which contained no observable flow (i.e., contained predominantly solid matrix) thereby leaving $\sim 80 \times 10^{3}$ voxels for which propagator data are shown. The mean and standard deviation of the local propagators were calculated using Eq. 9 and 10 and plotted in Fig. 5a and b, respectively. The result for each pair of local propagators is represented by a single point. Ideally, all points should lie on the red diagonal line, in which case the statistics for the CS and FS results are identical. Excellent agreement is indeed observed. A small deviation from the diagonal can be seen in Fig. 5a only at higher mean displacements, where the mean displacement from the CS-reconstructed local propagators is lower than the mean displacement derived from the fully-sampled local propagators. The data shown in Fig. 5 confirm that the statistical moments obtained from the spatially-resolved propagators can be used to characterise the 3D flow field in heterogeneous porous media.

\subsection{Spatially-resolved propagators within the carbonate rock core}

The characteristics of the 3D spatially-resolved propagators acquired for the carbonate rock core are shown in Fig. 6. In Fig. 6a, a comparison of the spatially-unresolved propagator obtained by the same four approaches as in Fig. 4a is shown. Although the zero-filled FT solution is clearly of lower quality, the agreement between the other propagators is very good. This result is relevant for two reasons. First, it is expected that within a rock, significant internal gradients and effects from the 
presence of paramagnetic impurities shorten the relaxation time significantly relative to the model system. However, the result in Fig. 6a, shows that the effects of relaxation can be kept to a minimum during the RARE pulse train. Furthermore, the superficial flow velocity derived from the spatiallyunresolved propagator is found to be $350 \mu \mathrm{m} \mathrm{s}^{-1}$, which compares well to the $389 \mu \mathrm{m} \mathrm{s}^{-1}$ calculated from the gravimetric porosity of the rock sample at the given flow rate. This result also shows that that total propagator adequately represents the flow within the rock despite the presence of internal gradients and relaxation sinks. Second, it shows that CS reconstruction of the spatially-resolved propagator also works well in the rock. This is particularly reassuring because the solid-liquid interfaces within the rock are not resolved as well as in the model system at the acquired spatial resolution, which could potentially have adversely affected the performance of the TV regularisation since TV promotes reconstruction of interfaces. For completeness it is noted that in terms of PSNR, the CS reconstruction $(66.6 \mathrm{~dB})$ of the spatially-resolved propagator is of significantly higher quality than the zero-filled FT solution $(52.5 \mathrm{~dB})$.

In Figure 6b-f, the 3D spatially-resolved propagator is again explored on the per-voxel level. Figure $6 \mathrm{~b}$ shows an $x, y$-slice through a 3D intensity image acquired at three times higher spatial resolution than the spatially-resolved propagator, i.e. $350 \mu \mathrm{m}$ isotropic spatial resolution. The structural heterogeneity across the rock is clearly observed. Four locations have been indicated with red circles, located at the tip of each arrowhead. The local propagators associated with voxels 1, 2, 3, and 4 are shown in Fig. 6c, d, e, and f, respectively. For each of the local propagators, a comparison is made between the fully-sampled FT, and the $12.5 \%$ sampled zero-filled FT and compressed sensing results. Because the structural features of the rock that underlie the apparent flow behaviour are often smaller than the spatial resolution, it is not possible to rationalise the shape of the propagator from the location of the voxel within the image, as was possible for the local propagators within the model system. As expected, the local propagators reveal a wide range of flow behaviour, ranging from nearstagnant to significant flow and dispersion. The mean displacement $\mu$ and standard deviation $\sigma$ of the propagators shown are 4 and $30 \mu \mathrm{m} ; 36$ and $77 \mu \mathrm{m} ; 225$ and $234 \mu \mathrm{m}$, and 207 and $212 \mu \mathrm{m}$ for voxels $1,2,3$, and 4 , respectively.

\section{Conclusions}

The opportunity to increase under-sampling when employing compressed sensing reconstruction when the dimensionality of the data is increased is exploited in acquiring 3D spatially-resolved propagators. The data are acquired as a 4-dimensional data set; with 3 spatial dimensions (k) and a displacement (q) dimension. Implementation of an under-sampled APGSTE-RARE experiment for acquiring 3D spatially-resolved propagators was described. It was shown that spatially-resolved propagators with 134,400 voxels, each containing a local propagator of 65 points, can be acquired using a sampling rate of $12.5 \%$, thereby reducing the acquisition time by almost an order of 
magnitude. A very high reconstruction quality was achieved for propagators acquired on a packed bed model system and on a carbonate rock, showing that still more under-sampling is possible whilst retaining an acceptable reconstruction quality.

\section{Acknowledgements}

The authors would like to thank Royal Dutch Shell plc for funding this work. 


\section{References}

[1] Gladden LF, Sederman AJ. Recent advances in Flow MRI. J Magn Reson 2013;229:2-11. doi:10.1016/j.jmr.2012.11.022.

[2] de Kort DW, Reci A, Ramskill NP, Appel M, de Jong H, Mantle MD, et al. Acquisition of spatially-resolved displacement propagators using compressed sensing APGSTE-RARE MRI. J Magn Reson 2018;295:45-56. doi:10.1016/j.jmr.2018.07.012.

[3] Scheenen TWJ, van Dusschoten D, de Jager PA, Van As H. Microscopic displacement imaging with pulsed field gradient turbo spin-echo NMR. J Magn Reson 2000;142:207-15. doi:10.1006/jmre.1999.1916.

[4] Scheenen TWJ, Vergeldt FJ, Windt CW, de Jager PA, Van As H. Microscopic imaging of slow flow and diffusion: A pulsed field gradient stimulated echo sequence combined with turbo spin echo imaging. J Magn Reson 2001;151:94-100. doi:10.1006/jmre.2001.2362.

[5] Shukla MN, Vallatos A, Phoenix VR, Holmes WM. Accurate phase-shift velocimetry in rock. J Magn Reson 2016;267:43-53. doi:10.1016/j.jmr.2016.04.006.

[6] Holland DJ, Gladden LF. Less is more: How compressed sensing is transforming metrology in chemistry. Angew Chem Int Ed 2014;53:13330-40. doi:10.1002/anie.201400535.

[7] Lustig M, Donoho DL, Santos JM, Pauly JM. Compressed sensing MRI. IEEE Signal Process Mag 2008;25:72-82. doi:10.1109/MSP.2007.914728.

[8] Cotts RM, Hoch MJR, Sun T, Markert JT. Pulsed field gradient stimulated echo methods for improved NMR diffusion measurements in heterogeneous systems. J Magn Reson (1969) 1989;83:252-66. doi:10.1016/0022-2364(89)90189-3.

[9] Hennig J, Nauerth A, Friedburg H. RARE imaging: A fast imaging method for clinical MR. Magn Reson Med 1986;3:823-33. doi:10.1002/mrm.1910030602.

[10] Gullion T, Baker DB, Conradi MS. New, compensated Carr-Purcell sequences. J Magn Reson 1990;89:479-84. doi:10.1016/0022-2364(90)90331-3.

[11] Carr HY, Purcell EM. Effects of diffusion on free precession in nuclear magnetic resonance experiments. Phys Rev 1954;94:630-8. doi:10.1103/physrev.94.630.

[12] Meiboom S, Gill D. Modified spin-echo method for measuring nuclear relaxation times. Rev Sci Instrum 1958;29:688-91. doi:10.1063/1.1716296.

[13] Ahola S, Perlo J, Casanova F, Stapf S, Blümich B. Multiecho sequence for velocity imaging in inhomogeneous rf fields. J Magn Reson 2006;182:143-51. doi:10.1016/j.jmr.2006.06.017.

[14] Ramskill NP, Bush I, Sederman AJ, Mantle MD, Benning M, Anger BC, et al. Fast imaging of laboratory core floods using 3D compressed sensing RARE MRI. J Magn Reson 2016;270:187-97. doi:10.1016/j.jmr.2016.07.017.

[15] Ramskill NP, Sederman AJ, Mantle MD, Appel M, de Jong H, Gladden LF. In situ chemically-selective monitoring of multiphase displacement processes in a carbonate rock using 3D magnetic resonance imaging. Transport Porous Med 2018;121:15-35. doi:10.1007/s11242-017-0945-6.

[16] Lustig M, Donoho D, Pauly JM. Sparse MRI: The application of compressed sensing for rapid MR imaging. Magn Reson Med 2007;58:1182-95. doi:10.1002/mrm.21391.

[17] Morozov VA. Regularization of incorrectly posed problems and the choice of regularization parameter. USSR Comput Math \& Math Phys 1966;6:242-51. doi:10.1016/00415553(66)90046-2.

[18] Chambolle A, Pock T. A first-order primal-dual algorithm for convex problems with Applications to Imaging. J Math Imaging Vis 2010;40:120-45. doi:10.1007/s10851010-0251-1.

[19] Chambolle A, Pock T. An introduction to continuous optimization for imaging. Acta Numer 2016;25:161-319. doi:10.1017/S096249291600009X.

[20] Benning M, Gladden L, Holland D, Schönlieb C-B, Valkonen T. Phase reconstruction from velocity-encoded MRI measurements - A survey of sparsity-promoting variational approaches. J Magn Reson 2014;238:26-43. doi:10.1016/j.jmr.2013.10.003. 
[21] Wapler MC, Leupold J, Dragonu I, Elverfeld von D, Zaitsev M, Wallrabe U. Magnetic properties of materials for MR engineering, micro-MR and beyond. J Magn Reson 2014;242:233-42. doi:10.1016/j.jmr.2014.02.005. 


\section{Figure captions}

Figure 1: Pulse sequence to acquire 3D spatially-resolved propagators, which consists of a 13interval APGSTE sequence followed by an XY-phase cycled RARE sequence. In the RARE sequence, an XY-16 phase cycle is used for the $180^{\circ}$ inversion pulses. Each successive pair of odd and even echoes is identically phase-encoded in $\mathbf{k}_{\text {phase }}$, so that two separate spatially-resolved propagators are acquired that can be added together during post-processing. The directions of displacement (q) and spatial (k) encoding can be chosen independently; in this work, the read direction and the direction of flow encoding were both parallel to the superficial flow direction $z$.

Figure 2: Sidelobe-to-peak ratio for a range of different pseudo-random $\mathbf{q}, \mathbf{k}_{\text {phase }}$-sampling patterns to be used with the APGSTE-RARE experiment, for different values of $N_{\text {ech }}$ and degrees of sampling: ( $)$ ) 25\%, ( $) 12.5 \%,(\nabla) 6.25 \%,(\Delta) 3.125 \%$. The patterns had sizes of $\mathbf{k}_{\text {phase }_{1}} \times \mathbf{k}_{\text {phase }_{2}} \times \mathbf{q}=40 \times 40 \times 65$ points, and were generated on the basis of a pdf as in Eq. 2 and 3 with $n_{\mathbf{k}_{\text {phase }}}, n_{\mathbf{q}}=7$ and with a fully-sampled spherical region of 4 points in diameter; 5,000 random sampling patterns were generated from which the pattern with the lowest SPR was selected. A lower SPR corresponds to greater incoherence in the sampled data.

Figure 3: Three cross-sections of the sampling patterns used in this study. The white pixels correspond to the positions at which a line in the readout dimension $\mathbf{k}_{\text {read }}$ is sampled fully. In (b) and (c) it can be seen that the patterns are symmetrical in the centre of $\mathbf{q}$-space to allow for addition of the odd and even echoes during post-processing.

Figure 4: Propagators for water flow through the packed bed of spheres and hollow cylinders (observation time $\Delta=200 \mathrm{~ms}$ ). (a) Comparison of the total displacement propagator in the packed bed obtained by four different methods: A spatially-unresolved APGSTE experiment (- - -); summation of the individual, per-voxel propagators from a fully-sampled, Fourier-transformed 3D spatiallyresolved experiment (-); summation of the individual, per-voxel propagators from a $12.5 \%$ sampled, zero-filled, and Fourier-transformed 3D spatially-resolved experiment (- - -); summation of the individual, per-voxel propagators from the same $12.5 \%$ sampled, 3D spatially-resolved experiment reconstructed using TV-regularised compressed sensing (- - -). (b) A transverse $(x, y)$ image slice taken from a 3D intensity image acquired at $350 \mu \mathrm{m}$ isotropic spatial resolution. Arrows indicate four voxels (1-4) for which local propagators are shown (c-f). A comparison is made between local propagators obtained from fully-sampled, Fourier-transformed data (-), with data acquired at $12.5 \%$ sampling followed by: zero-filling and Fourier transformation (-.-), and the TVregularised CS reconstruction (- - -). 
Figure 5: Voxel-by-voxel comparison of (local) propagator moments calculated for the fully-sampled (FS), Fourier-transformed data and the compressed sensing (CS) reconstruction. Shown are comparisons of the (a) mean $(\mu)$ and (b) standard deviation $(\sigma)$; each dot represents one of 79,845 local propagators. The red line is the diagonal, shown for reference.

Figure 6: Propagators for water flow through the Edwards Brown carbonate rock plug (observation time $\Delta=200 \mathrm{~ms}$ ). (a) Comparison of the total displacement propagator in the packed bed obtained by four different methods: A spatially-unresolved APGSTE experiment (- - ); summation of the individual, per-voxel propagators from a fully-sampled, Fourier-transformed 3D spatially-resolved experiment (-); summation of the individual, per-voxel propagators from a $12.5 \%$ sampled, zerofilled, and Fourier-transformed 3D spatially-resolved experiment (-•-); summation of the individual, per-voxel propagators from the same $12.5 \%$ sampled, 3D spatially-resolved experiment reconstructed using TV-regularised compressed sensing (- - -). (b) A transverse $(x, y)$ image slice taken from a 3D intensity image acquired at $350 \mu \mathrm{m}$ isotropic spatial resolution. Arrows indicate four voxels $(1-4)$ for which local propagators are shown (c-f). A comparison is made between local propagators obtained from fully-sampled, Fourier-transformed data (-), with data acquired at $12.5 \%$ sampling followed by: zero-filling and Fourier transformation (-•-), and the TV-regularised CS reconstruction (- - -). 


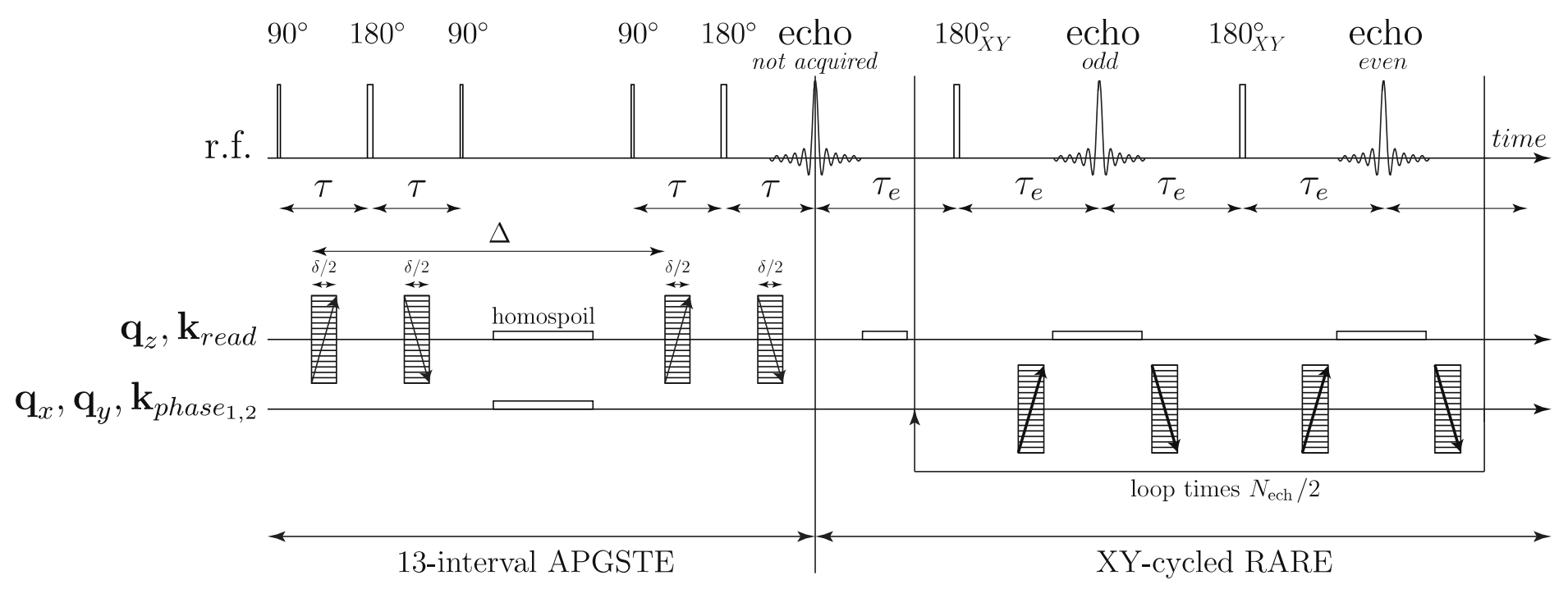

Figure 1 


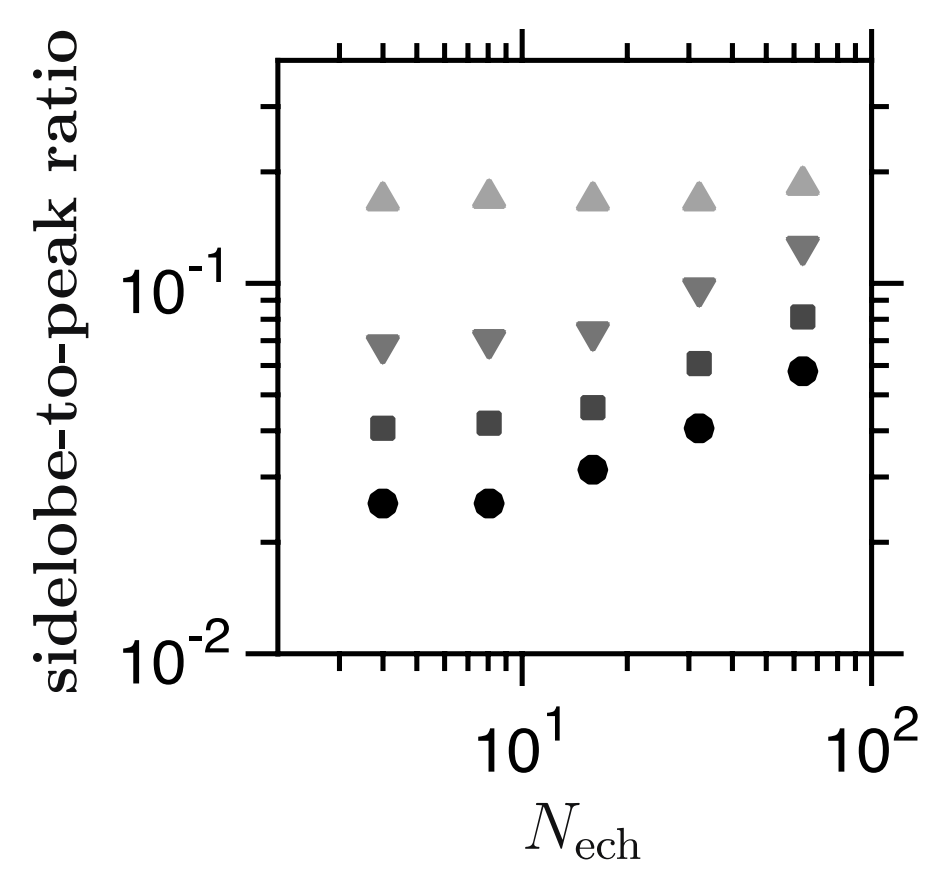

Figure 2 
(a)

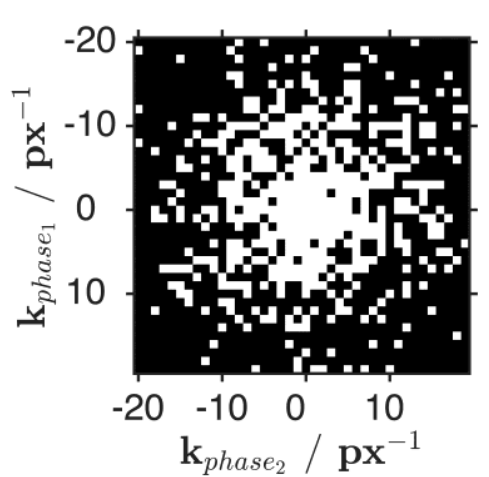

(b)

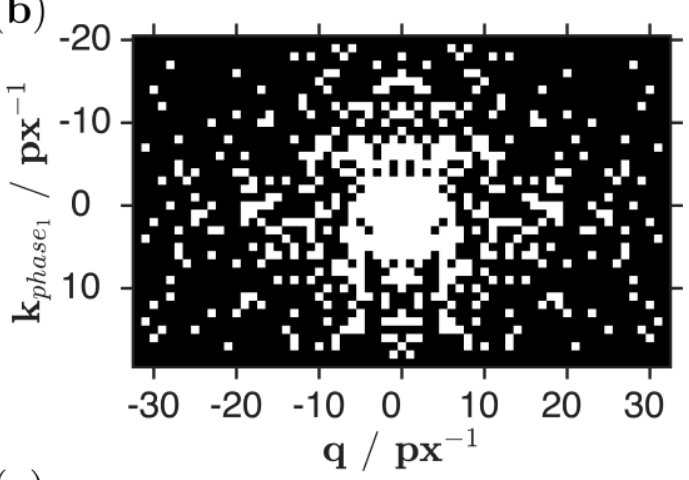

(c)

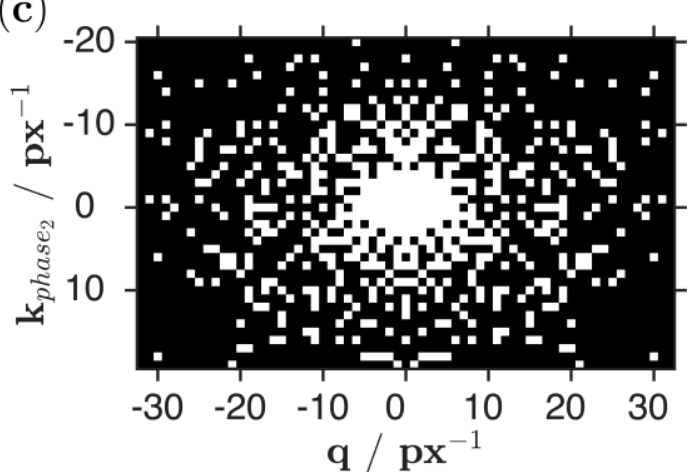

Figure 3 

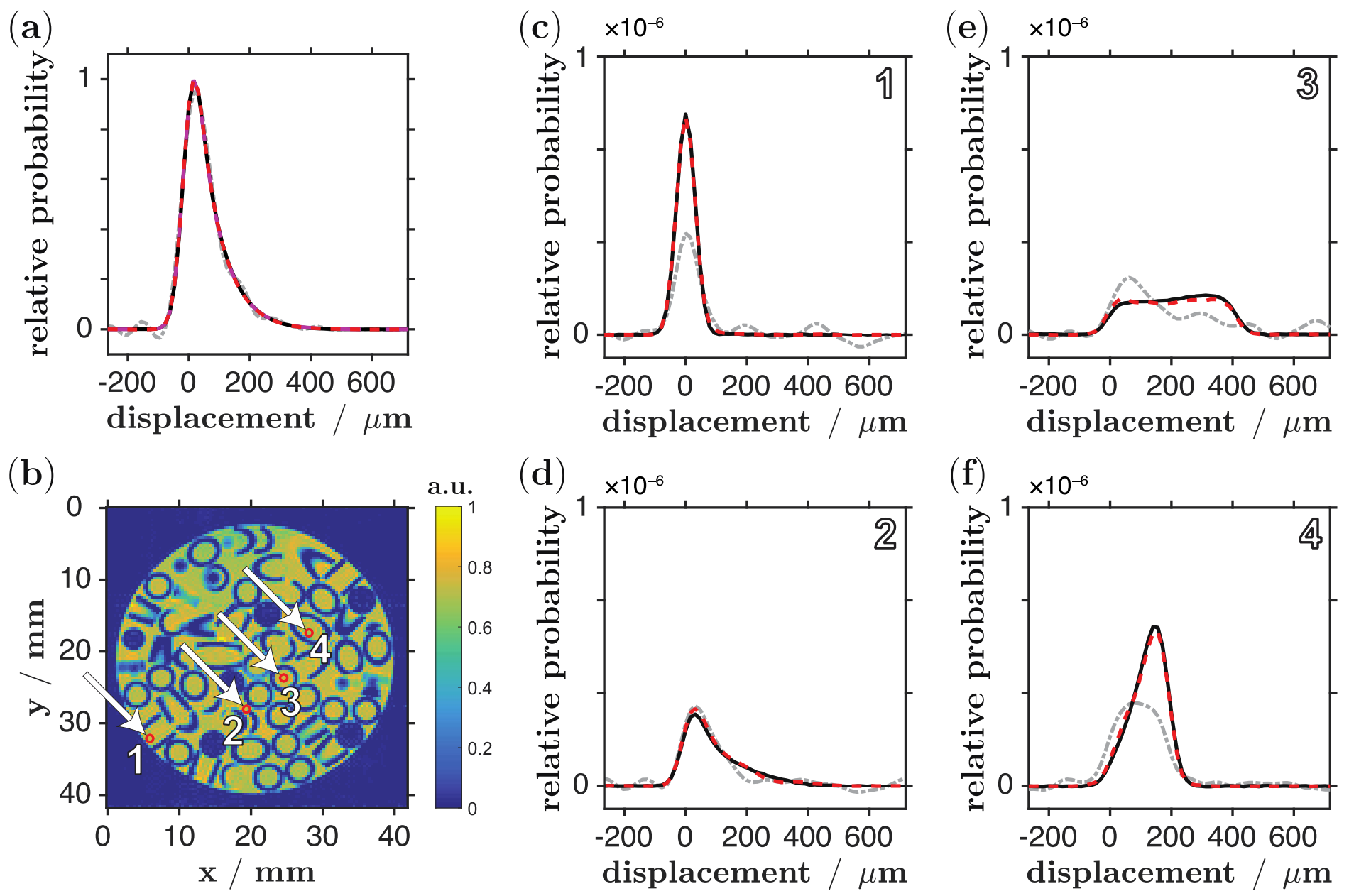

Figure 4 

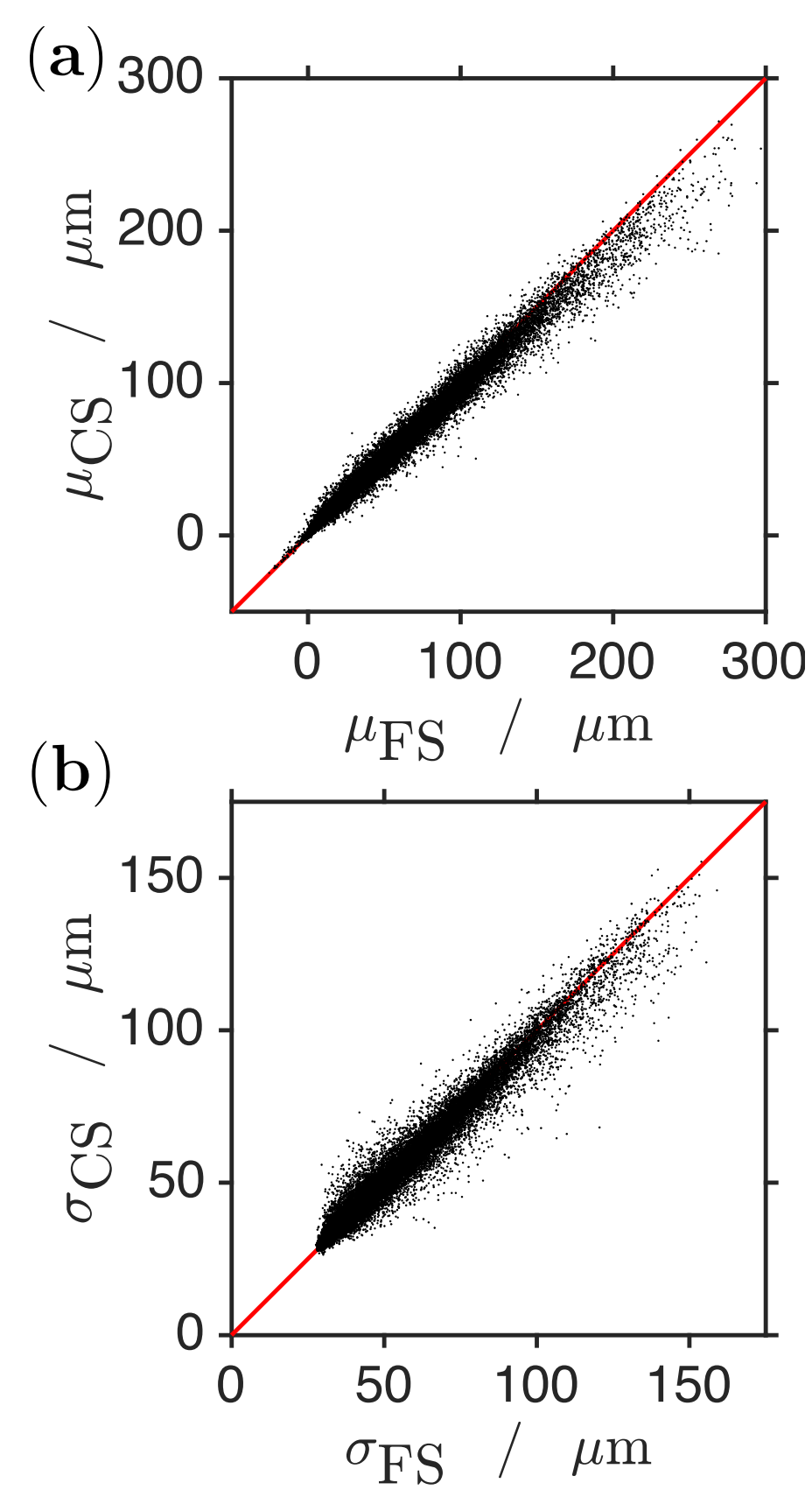

Figure 5 


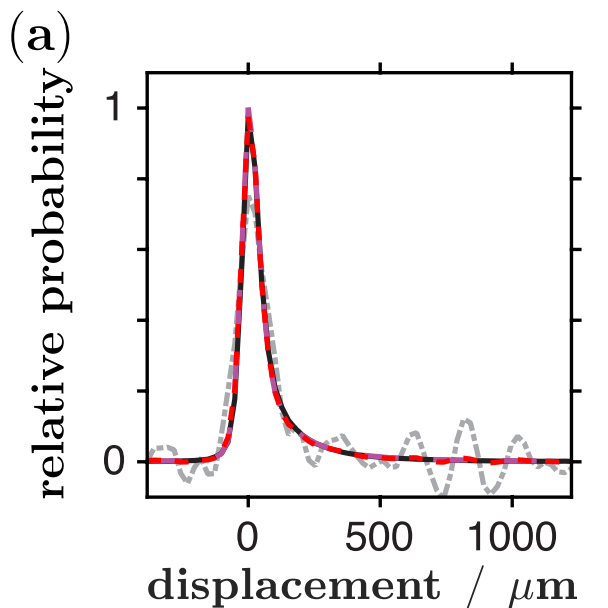

(b)

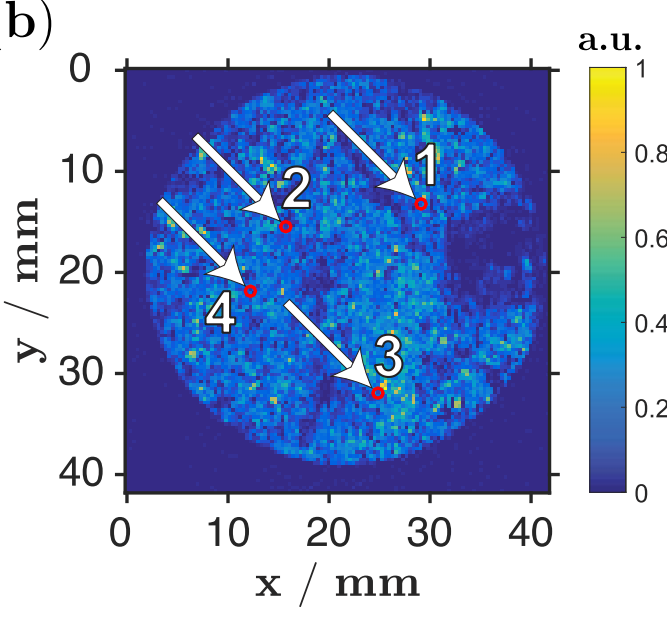

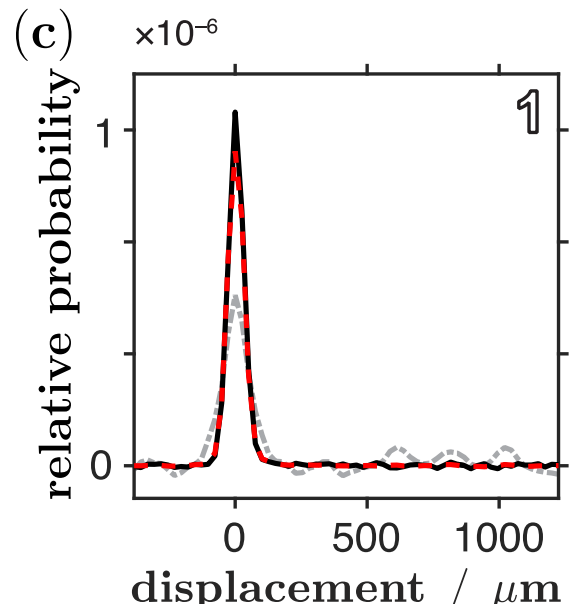

(d)

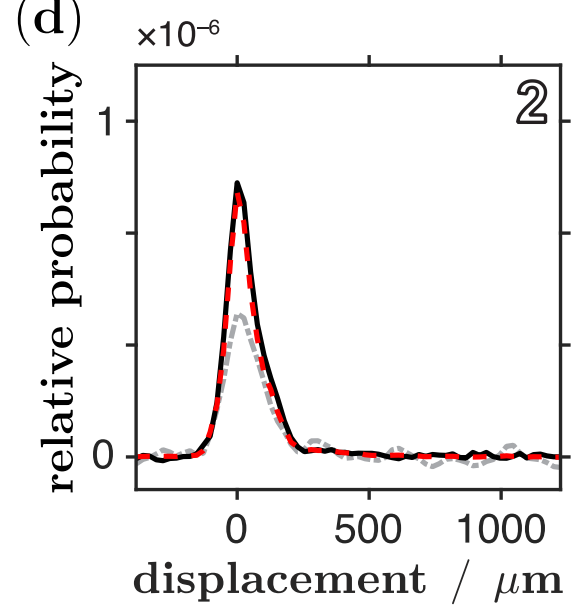

(e) $\times 10^{-6}$

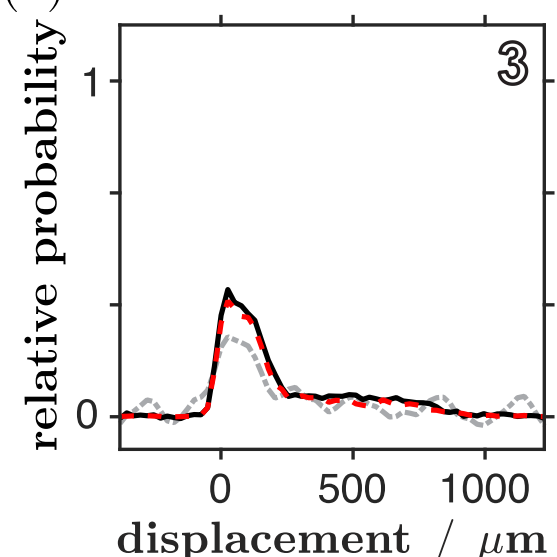

(f) $\times 10^{-6}$

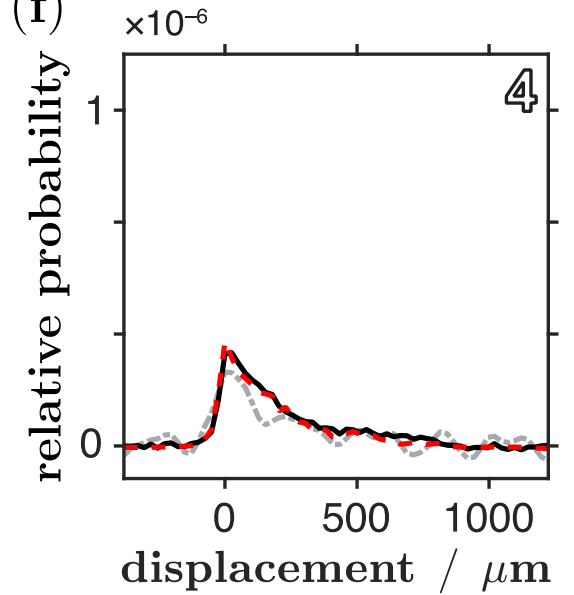

Figure 6 